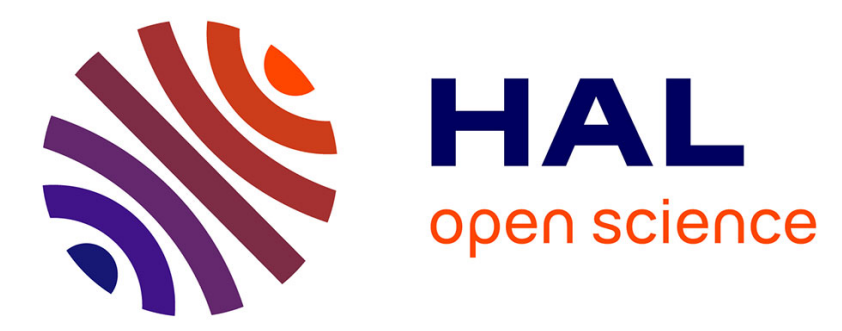

\title{
Ankle, hip and stepping strategies for humanoid balance recovery with a single Model Predictive Control scheme
}

\author{
Zohaib Aftab, Thomas Robert, Pierre-Brice Wieber
}

\section{To cite this version:}

Zohaib Aftab, Thomas Robert, Pierre-Brice Wieber. Ankle, hip and stepping strategies for humanoid balance recovery with a single Model Predictive Control scheme. IEEE-RAS International Conference on Humanoid Robots (Humanoids 2012), Nov 2012, Osaka, Japan. pp.159-164, 10.1109/HUMANOIDS.2012.6651514 . hal-02487619

\section{HAL Id: hal-02487619 \\ https://hal.inria.fr/hal-02487619}

Submitted on 21 Feb 2020

HAL is a multi-disciplinary open access archive for the deposit and dissemination of scientific research documents, whether they are published or not. The documents may come from teaching and research institutions in France or abroad, or from public or private research centers.
L'archive ouverte pluridisciplinaire HAL, est destinée au dépôt et à la diffusion de documents scientifiques de niveau recherche, publiés ou non, émanant des établissements d'enseignement et de recherche français ou étrangers, des laboratoires publics ou privés. 


\title{
Ankle, hip and stepping strategies for humanoid balance recovery with a single Model Predictive Control scheme
}

\author{
Zohaib Aftab, Thomas Robert and Pierre-Brice Wieber
}

\begin{abstract}
While humans are highly efficient in dealing with balance perturbations, current biped humanoid robots are far from showing similar skills. This is mainly due to the limited capacity of current robot controllers to deal with the inherently complex dynamics of biped robots. Though Model Predictive Control schemes have shown improved robustness to perturbations, they still suffer from a few shortcomings such as not considering the upper body inertial effects or nonoptimal step durations. We propose here a Model Predictive Control scheme that specifically addresses these shortcomings and generates human-like responses to perturbations, involving appropriate combinations of ankle, hip and stepping strategies, with automatically adjusted step durations. The emphasis of this paper is on modeling and analyzing the effects of different cost functions and coefficients on the behavior of the controller while leaving real-time implementations and experiments for later work.
\end{abstract}

\section{INTRODUCTION}

Recovering balance after a perturbation occurs can be a complex process involving different strategies depending on the situation. Experimental study of this process in humans has led to identify three basic strategies, the ankle, hip and stepping strategies [1] , [2]. It has been observed that the ankle and hip strategies work in parallel to recover balance, the amplitude of the hip strategy being positively scaled with the amplitude of the perturbation [3], [4]. The stepping strategy is only triggered in case of stronger perturbations, but below the theoretical stability limit of the previous strategies, so that appropriate combinations of the three strategies are generated in order to recover effectively with suitable step length and duration.

Current biped robots are still far from showing similar versatile and efficient responses to perturbations. Although the hip strategy has been demonstrated to considerably enhance the stability of biped systems through the use of the upper-body inertia [5], [6] most biped control schemes only implement the ankle strategy, only regulating the Center of Pressure (CoP) with feet positions fixed in advance [7], [8], [9]. Stepping strategies have already been addressed with the Capture Point and similar concepts [10], [11]], [112], [13], [114] or with a Linear Model Predictive Control (LMPC) scheme including future steps as potential control variables [15].

But all of these schemes suffer from the same common limitations:

Z. Aftab and T. Robert are with the IFSTTAR - Université Lyon 1, LBMC UMR T9406, France. P.-B. Wieber is with the Bipop team at INRIA Rhône-Alpes, France. zohaib.aftabdifsttar.fr, thomas.roberteifsttar.fr, pierre-brice.wiebereinria.fr

The work of $Z$. Aftab is supported by a doctoral research allocation from the Rhône-Alpes region.
- when the hip strategy is considered, a sequential transition from ankle to hip strategy is usually implied by saturation or arbitrary thresholding [16], [17], [18],

- the step duration, which has a strong impact on the efficiency of the balance recovery, is always estimated or fixed in advance, which can be seriously limiting,

- except for [10] and the LMPC scheme [15], all the stepping strategies mentioned earlier require that the biped system stops in exactly one step, what is neither always feasible nor desirable.

Our goal here is to propose an efficient balance recovery scheme implementing an appropriate combination of ankle, hip and stepping strategies. Such a combination has already been obtained in simulation in [19] and the results were impressive, but with a heuristic approach lacking both the provable stability and the optimality that we are looking for with our LMPC scheme. A similar combination has also been discussed in [20], but without much details ("distribute the high frequency components to the flywheel and the low frequency components to the inverted pendulum") and therefore without much analysis.

Of all the balance controllers discussed above, the LMPC scheme proposed in [15] is the most promising technique in our view since it deals naturally with multiple step strategies thanks to the calculations over a future horizon of time. We will use it therefore as our main building block, and amend it to offset its different shortcomings exposed above, namely:

- we will integrate upper body inertial effects in order to generate appropriate combinations of hip strategies with the ankle and stepping strategies already appearing in [15],

- we will integrate a proper optimization of the step duration for improved efficiency of the balance recovery behavior.

We are going to present this amended MPC scheme in the next Section, analyze the optimization of the step duration in Section $\mathbb{}$ and demonstrate in Section $\mathbb{\text { IV }}$ how the ankle, hip and stepping strategies are eventually combined to generate efficient balance recovery behaviors.

\section{AN MPC SCHEME FOR BALANCE RECOVERY}

\section{A. Dynamic model and prediction horizon}

A well regarded simple model of human and humanoid balance is the Linear Inverted Pendulum (LIP) where the whole mass $m$ of the system is considered concentrated at the Center of Mass (CoM), moving at a constant height $h$ above the ground [21]. Since we're also interested here in 
the effect of trunk rotations on balance, we supplement this LIP model with a simple flywheel model as in [5] and [22], leading to the simple linear dynamics

$$
m h \ddot{c}+j \ddot{\theta}=m g(c-z)
$$

where $c$ and $z$ are the horizontal coordinates of the CoM and CoP, $\theta$ is the orientation of the trunk, $j$ the inertia of the trunk and $g$ the norm of the gravity force.

The controller design initially proposed in [23] and further refined in [15] and [24] is based on anticipating future motions on a prediction horizon composed of $N$ time intervals of equal length $T$. The motion on this future horizon is considered having a piecewise constant third derivative $\dddot{c}$ and $\dddot{\theta}$ over each time interval. Considering the state

$$
\hat{c}_{k}=\left[\begin{array}{c}
c_{k} \\
\dot{c}_{k} \\
\ddot{c}_{k}
\end{array}\right], \hat{\theta}_{k}=\left[\begin{array}{c}
\theta_{k} \\
\dot{\theta}_{k} \\
\ddot{\theta}_{k}
\end{array}\right]
$$

of the system at a time $t_{k}$, all it takes is a straightforward time integration to relate the position, speed and acceleration of the CoM over the whole prediction horizon

$$
C_{k+1}=\left[\begin{array}{c}
c_{k+1} \\
\vdots \\
c_{k+N}
\end{array}\right], \dot{C}_{k+1}=\left[\begin{array}{c}
\dot{c}_{k+1} \\
\vdots \\
\dot{c}_{k+N}
\end{array}\right], \ddot{C}_{k+1}=\left[\begin{array}{c}
\ddot{c}_{k+1} \\
\vdots \\
\ddot{c}_{k+N}
\end{array}\right]
$$

to the piecewise constant third derivative

$$
\dddot{C}_{k+1}=\left[\begin{array}{c}
\dddot{c}_{k+1} \\
\vdots \\
\dddot{c}_{k+N}
\end{array}\right]
$$

through simple matrices:

$$
\begin{aligned}
C_{k+1} & =S_{p} \hat{c}_{k}+U_{p} \dddot{C}_{k}, \\
\dot{C}_{k+1} & =S_{v} \hat{c}_{k}+U_{v} \dddot{C}_{k}, \\
\ddot{C}_{k+1} & =S_{a} \hat{c}_{k}+U_{a} \dddot{C}_{k} .
\end{aligned}
$$

Detailed formulas for these matrices can be found in [15]. Identical relationships obviously hold for the trunk rotation $\theta$.

The linear dynamics (UI) can be reversed to compute the position of the CoP from the motion of the human or humanoid system:

$$
z=c-\frac{h}{g} \ddot{c}-\frac{j}{m g} \ddot{\theta} .
$$

As before, we can easily relate the position of the CoP over the whole prediction horizon

\begin{tabular}{|c|c|c|c|}
\hline Variable & Symbol & Value & Ref. \\
\hline Body height & $H$ & $1.76 \mathrm{~m}$ & \\
\hline Body mass & $m$ & $76 \mathrm{~kg}$ & \\
\hline CoM height & $h=0.575 \times H$ & $1.012 \mathrm{~m}$ & {$[25]$} \\
\hline Foot length & $0.152 \times H$ & $0.268 \mathrm{~m}$ & [25] \\
\hline Max trunk rotation & $\theta_{\max }$ (forward) & $\pi / 2 \mathrm{rad}$ & \\
\hline Min trunk rotation & $\theta_{\min }$ (backward) & $-\pi / 3 \mathrm{rad}$ & \\
\hline Trunk inertia & $j$ & $8 \mathrm{~kg} \cdot \mathrm{m}^{2}$ & {$[25]$} \\
\hline Max hip torque & $\tau_{\max }$ & 190 N.m & [26] \\
\hline Control weight & $\alpha_{1}$ & $1 \mathrm{~m} \cdot \mathrm{s}^{-1}$ & \\
\hline Control weight & $\alpha_{2}$ & $10 \mathrm{rad}_{\mathrm{s}} \mathrm{s}^{-1}$ & \\
\hline Control weight & $\alpha_{3}$ & $200 \mathrm{~m} \cdot \mathrm{s}^{-2}$ & \\
\hline Control weight & $\alpha_{4}$ & $100 \mathrm{~m} \cdot \mathrm{s}^{-3}$ & \\
\hline Control weight & $\alpha_{5}$ & 300 rad.s $^{-3}$ & \\
\hline Control weight & $\alpha_{6}$ & $100 \mathrm{~m}$ & \\
\hline
\end{tabular}

$$
Z_{k+1}=\left[\begin{array}{c}
z_{k+1} \\
\vdots \\
z_{k+N}
\end{array}\right]
$$

to the piecewise constant third derivatives $\dddot{C}_{k}$ and $\dddot{\Theta}_{k}$ through simple matrices:

$$
Z_{k+1}=S_{z}\left[\begin{array}{c}
\hat{c}_{k} \\
\hat{\theta}_{k}
\end{array}\right]+U_{z}\left[\begin{array}{c}
\dddot{C}_{k} \\
\dddot{\Theta}_{k}
\end{array}\right]
$$

TABLE I: Anthropomorphic proportions used in the simulation

with

$$
\begin{aligned}
S_{z} & =\left[\begin{array}{ll}
S_{p}-\frac{h}{g} S_{a} & -\frac{j}{m g} S_{a}
\end{array}\right], \\
U_{z} & =\left[\begin{array}{ll}
U_{p}-\frac{h}{g} U_{a} & -\frac{j}{m g} U_{a}
\end{array}\right] .
\end{aligned}
$$

\section{B. Kinematic and dynamic constraints}

The dynamics (II) of human and humanoid balance is subject to a series of kinematic and dynamic constraints that have to be satisfied over the whole prediction horizon, for all $i \in[k+1, \ldots k+N]$. For example, trunk rotation is limited by articulation constraints. With such a simple model, it will be constrained by direct bounds

$$
\theta_{\min } \leq \theta_{i} \leq \theta_{\max }
$$

The same concerning hip torques:

$$
j\left|\ddot{\theta}_{i}\right| \leq \tau_{\max }
$$

(values for all the geometric and dynamic parameters of the model are gathered in Table II).

If $f_{i}$ represents the horizontal position of the support foot on the ground at time $t_{i}$, the maximum extension of the support leg can be simply enforced with the horizontal position of the CoM:

$$
\left\|c_{i}-f_{i}\right\| \leq l_{\max }
$$

If $f_{i}^{\prime}$ represents the horizontal position of the swing foot above the ground, its acceleration will also be bounded:

$$
\left\|\ddot{f}_{i}^{\prime}\right\| \leq \ddot{f}_{\max }^{\prime}
$$

Finally, the $\operatorname{CoP} z$ is also constrained to stay within the boundaries of the support polygon, what can be expressed in the following way:

$$
D\left(z_{i}-f_{i}\right) \leq b
$$

where $D$ and $b$ are a matrix and a vector encoding the shape of the foot with respect to the position $f_{i}$ of the support foot on the ground (more details can be found in [24]). 
In order to express these constraints over the whole prediction horizon, we have to relate the position of the support foot over the whole prediction horizon

$$
F_{k+1}=\left[\begin{array}{c}
f_{k+1} \\
\vdots \\
f_{k+N}
\end{array}\right]
$$

with the current support foot position $f_{k}$ which is fixed on the ground and the positions $\bar{F}_{k+1}$ of the future steps which will have to be decided by the balance controller. This can be done easily with matrices $V_{k+1}$ and $\bar{V}_{k+1}$ filled with 0 s and $1 \mathrm{~s}$ simply indicating which sampling times $t_{i}$ fall within which steps (more details can be found in [24]):

$$
F_{k+1}=V_{k+1} f_{k}+\bar{V}_{k+1} \bar{F}_{k+1} .
$$

\section{Controller design}

Following the controller design proposed in [15] and [24], we consider an MPC scheme that anticipates future motions over a prediction horizon. We are interested in regulating the balance of a humanoid system standing still and experiencing perturbations of various amplitude. The basic objective is therefore to minimize the speed $\dot{C}_{k+1}$ of the CoM and the rotation speed $\dot{\Theta}_{k+1}$ of the trunk over the prediction horizon while taking care of all the constraints listed earlier. Minimizing the acceleration $\ddot{F}_{k+1}^{\prime}$ of the swing foot will appear to be valuable as well in the next Section, leading to a multi-objective controller minimizing a weighted sum

$$
\min \frac{1}{\alpha_{1}^{2}}\left\|\dot{C}_{k+1}\right\|^{2}+\frac{1}{\alpha_{2}^{2}}\left\|\dot{\Theta}_{k+1}\right\|^{2}+\frac{1}{\alpha_{3}^{2}}\left\|\ddot{F}_{k+1}^{\prime}\right\|^{2} .
$$

But following the analysis developed in [15], the resulting motions appear to be significantly improved when minimizing also the third derivatives $\dddot{C}_{k}$ and $\dddot{\Theta}_{k}$ of the motion and the distance between the position $Z_{k+1}$ of the CoP and the center $F_{k+1}$ of the support foot. The final multi-objective controller looks therefore to minimize

$$
\begin{aligned}
& \min \frac{1}{\alpha_{1}^{2}}\left\|\dot{C}_{k+1}\right\|^{2}+\frac{1}{\alpha_{2}^{2}}\left\|\dot{\Theta}_{k+1}\right\|^{2}+\frac{1}{\alpha_{3}^{2}}\left\|\ddot{F}_{k+1}^{\prime}\right\|^{2} \\
& +\frac{1}{\alpha_{4}^{2}}\left\|\dddot{C}_{k}\right\|^{2}+\frac{1}{\alpha_{5}^{2}}\left\|\dddot{\Theta}_{k}\right\|^{2}+\frac{1}{\alpha_{6}^{2}}\left\|Z_{k+1}-F_{k+1}\right\|^{2},
\end{aligned}
$$

with weights $\alpha_{1}$ to $\alpha_{6}$ chosen with respect to the magnitude of the associated terms and their importance in the balance recovery process. Typical values are given in Table II.

As noted in [15], the third derivatives are weakly penalized (relatively high weights $\alpha_{4}$ and $\alpha_{5}$ ) only to smooth the resulting motion, with relatively low impact on the final behavior of the robot. Same thing with the final term and weight $\alpha_{6}$ which loosely centers the $\mathrm{CoP}$ in the support polygon. More important is the penalization of the trunk rotation speed with respect to the CoM speed, which affects how much trunk rotation is used. For a reference weight $\alpha_{1}$ of $1 \mathrm{~m} . \mathrm{s}^{-1}$, reasonable values for $\alpha_{2}$ are around 3 to 30 rad.s ${ }^{-1}$. Most important in this paper is the weight $\alpha_{3}$, the effect of which will be analyzed in more depths in the following Sections.

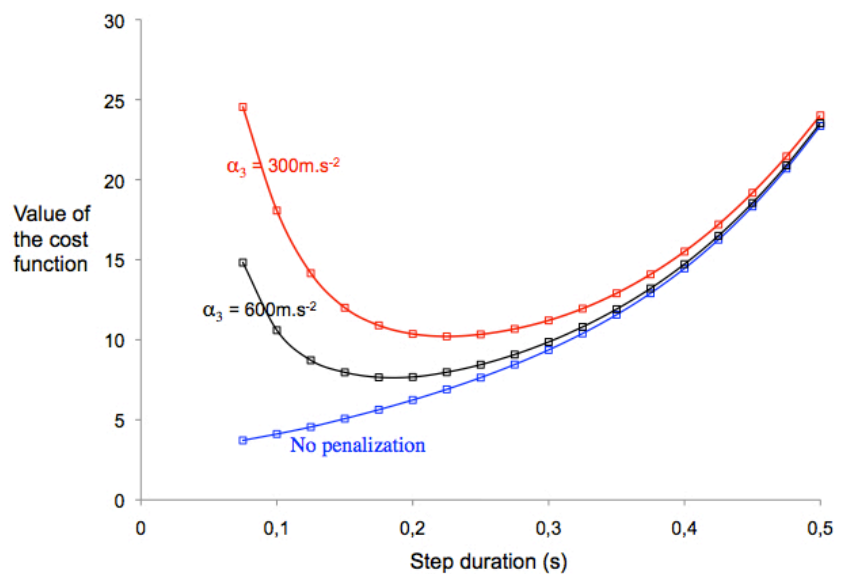

Fig. 1: Minimal value of the objective function for different penalization coefficients $\alpha_{3}$, or for no penalization at all of the swing foot acceleration $\ddot{F}_{k+1}^{\prime}$, for a given perturbation and different fixed step durations.

Since this controller only deals with the cartesian positions of the CoM and feet and orientation of the trunk, mapping these variables with the whole body motion of the articulated robot is done with classical Forward and Inverse Kinematics methods. The motion of the feet is interpolated with 5th degree polynomials between their current position, velocity and acceleration and their desired position on the ground with zero velocity and acceleration (no impact).

In the following Sections, this multi-objective controller will be considered only in the sagittal plane, with a prediction horizon of length $1 \mathrm{~s}(\mathrm{~N}=20, \mathrm{~T}=0.05 \mathrm{~s})$. The situation will be that of a human sized model standing still with both legs aligned in the sagittal plane, experiencing impact disturbances of varying amplitude.

\section{REgUlating THE STEP DURATION}

Recovering from perturbations with automatic step placement has already been realized successfully in [27], [15] and [24] with MPC schemes similar to the one presented here, but always with a fixed step duration. Yet, the adaptation of the step duration is at least as important as the adaptation of the step placement when having to recover from perturbations. Allowing the duration of the steps to vary has been tried in [28], but the steps ended up being always chosen the quickest possible: if no explicit lower limit was set on their duration, they were chosen to be infinitely quick!

The reason for this ill behavior lies in the objective function which was the same as the one proposed here in (2I), but without penalizing the acceleration $\ddot{F}_{k+1}^{\prime}$ of the swing foot. Let's have a look at the value of this objective function as a function of step duration in Fig. $⿴$ l for a postimpact CoM velocity of $1 \mathrm{~m} . \mathrm{s}^{-1}$. We can observe that when the acceleration of the swing foot is not penalized, this objective function decreases continuously with shorter step durations: quicker steps always allow minimizing CoM and trunk rotation speeds more and more efficiently. But quicker 


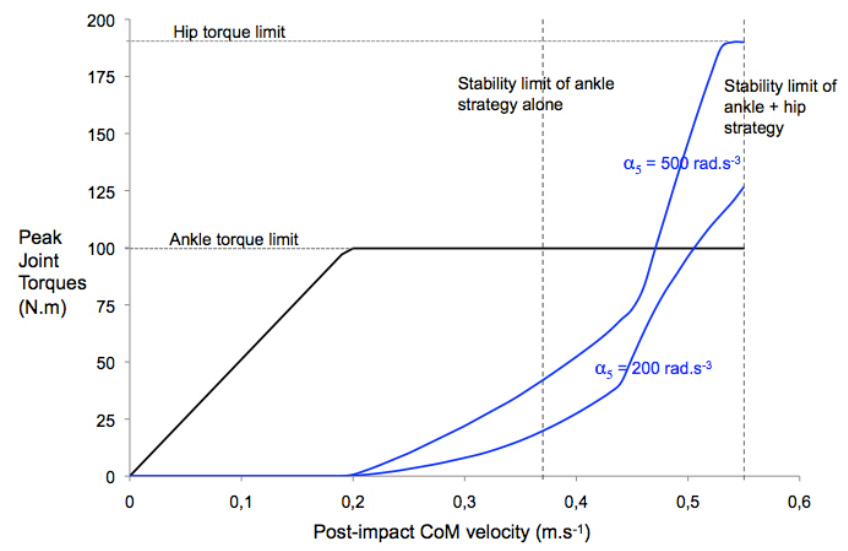

Fig. 2: Combination of ankle and hip strategies when feet are kept on the ground (no stepping). The disturbance is characterized by the post-impact CoM velocity. Resulting recovery response is plotted in terms of peak ankle and hip torques.

steps incur a mechanical and energetic cost [29], [30] which should be put in balance with the balance recovery objective.

We propose here to introduce a simple model of this mechanical cost in the objective function (2II) by penalizing the acceleration $\ddot{F}_{k+1}^{\prime}$ of the swing foot. We consider moreover in (16) that this acceleration can't anyway exceed a given maximum value. We can see in Fig. W that this combined objective function rises steeply for very quick steps: its minimum is now reached for intermediate step durations, which will depend on the coefficient $\alpha_{3}$. For the example depicted in Fig. आ, minimizing the combined objective function (2II) with a coefficient $\alpha_{3}$ equal to $300 \mathrm{~m} \cdot \mathrm{s}^{-2}$ selects a step duration of $0.23 \mathrm{~s}$.

Note that allowing the duration of the steps to vary makes the optimization problem (2II) become nonlinear, requiring more complex numerical solvers. In the case of an MPC scheme like here, this nonlinear optimization problem needs to be solved at high frequency for proper feedback efficiency since it is evolving with time as the current state of the system is evolving. Hopefully, parametric Sequential Quadratic Programming (SQP) algorithms allow to solve such evolving nonlinear problems at a relatively low computational cost [31]. Initial numerical experiments showed that we could solve the nonlinear MPC scheme presented here in less than $10 \mathrm{~ms}$, with room for improvement, so real-time implementations are largely within reach [28]. But we propose to focus here on the modeling issue, analyzing the effects of different cost functions and coefficients on the behavior of the controller, leaving real-time implementations and experiments for later work.

\section{Multiple Balance Recovery strategies}

Let's analyze first how the ankle and hip strategies combine in our controller when the feet are kept on the ground (no stepping) and the system is exposed to impacts of various magnitudes. We can see in Figure \ that for small perturbations, the ankle strategy is used increasingly until

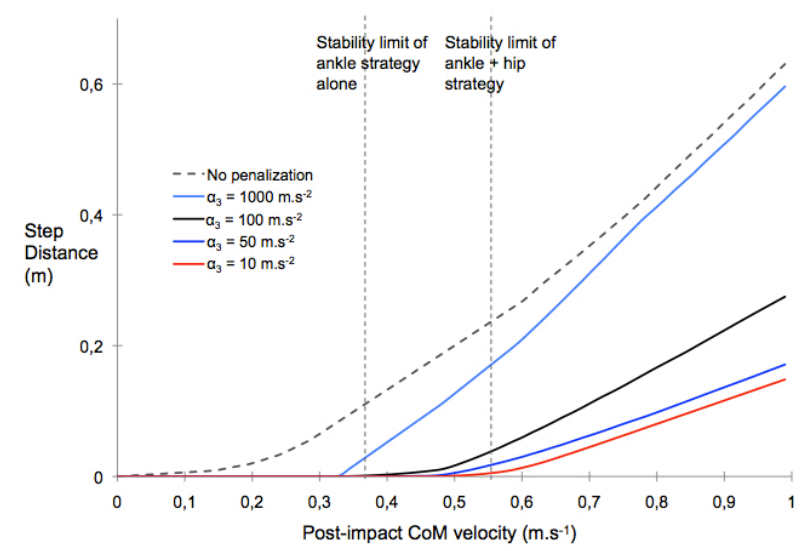

Fig. 3: Step length when reacting to varying post-impact CoM velocities for different penalization coefficients $\alpha_{3}$, or for no penalization at all of the swing foot acceleration $\ddot{F}_{k+1}^{\prime}$. The step duration is fixed to $0.3 \mathrm{~s}$.

it reaches its limit torque. If this ankle strategy is used alone, a stability limit is reached for a post-impact CoM velocity of approximately $0.37 \mathrm{~m} . \mathrm{s}^{-1}$. When the hip strategy is combined with the ankle strategy, they work in parallel to recover balance even if the stability limit of the ankle strategy alone hasn't been reached yet, and the amplitude of the hip strategy is positively scaled with the amplitude of the perturbation, similarly to what has been observed in humans [3], [4]. Together, these two strategies allow recovering from much stronger perturbations, with postimpact CoM velocities up to $0.55 \mathrm{~m} . \mathrm{s}^{-1}$.

Let's complete now the balance recovery behavior with the stepping strategy. In order to analyze how the three strategies combine, let's consider first a situation where the step duration is fixed to $0.3 \mathrm{~s}$. We can see in Figure [3] that if the acceleration $\ddot{F}_{k+1}^{\prime \prime}$ of the swing foot is not penalized, the stepping strategy is initiated even for the smallest perturbations, what isn't a reasonable behavior. This parallels the discussion we already had in Section III: if the stepping strategy incurs no cost, then there's no reason not to put it at work, even when it's absolutely not necessary nor even particularly helpful.

Depending on how much this acceleration is penalized, the stepping strategy will be activated at different levels of perturbations, as we can see once again in Figure [3. For a small penalization $\left(\alpha_{3}=1000 \mathrm{~m} \cdot \mathrm{s}^{-2}\right)$, the stepping strategy is initiated before even reaching the stability limit of the ankle strategy alone. For a strong penalization $\left(\alpha_{3}=10 \mathrm{~m} . \mathrm{s}^{-2}\right)$, it is initiated only when reaching the stability limit of the ankle and hip strategies working together. We can tune therefore very easily when the decision is taken to use the stepping strategy, and how much.

Let's combine now these three strategies with the regulation of the step durations and consider a situation where our model is standing still, upright while a force of 1.5 times its weight is applied horizontally forward during $0.05 \mathrm{~s}$ at the level of its CoM. All parameters are set according to 

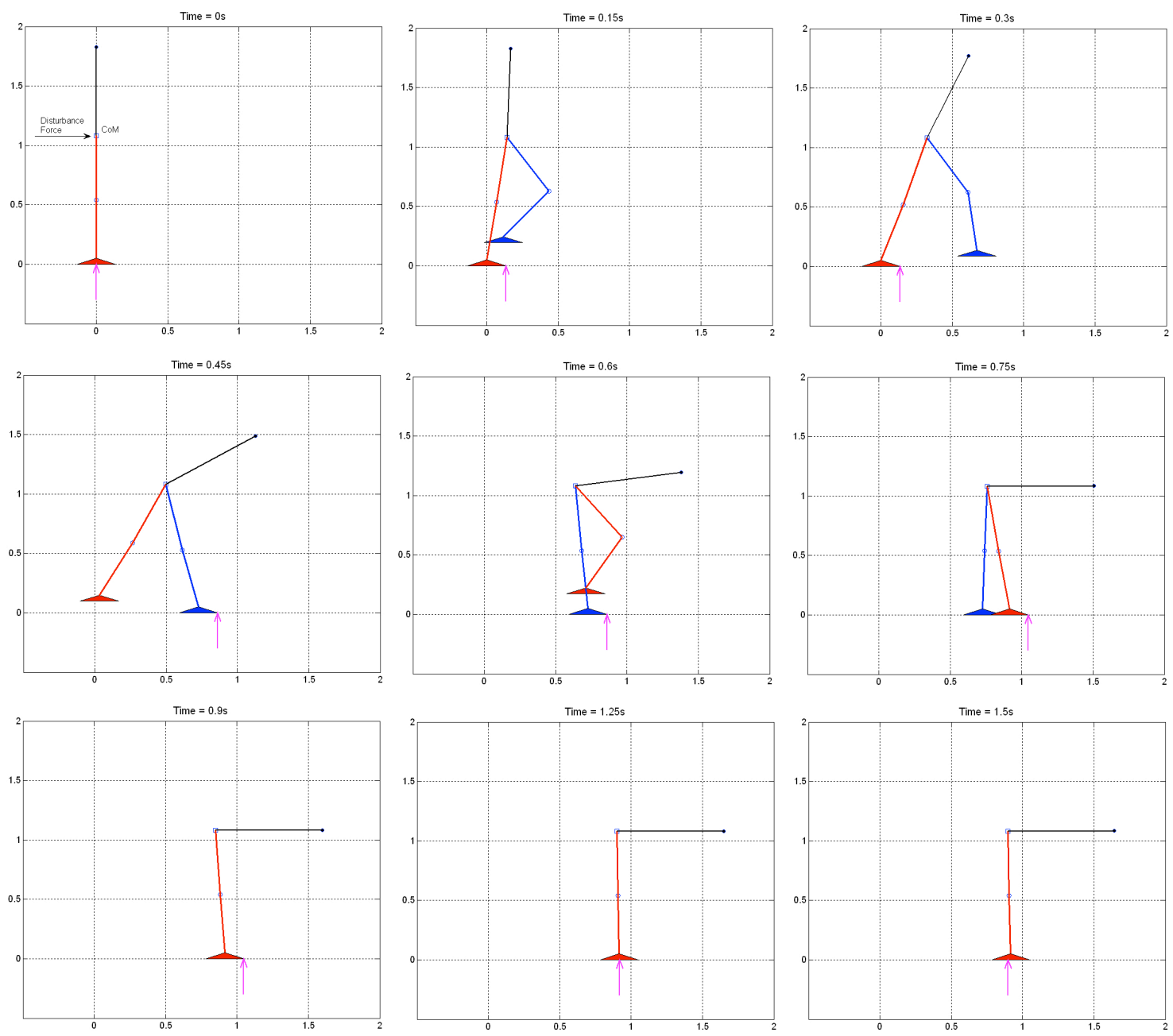

Fig. 4: Snapshots of a balance recovery motion combining ankle, hip and stepping strategies after a force of 1.5 times its weight applies horizontally forward during $0.05 \mathrm{~s}$ at the level of the CoM. Position of the CoP is indicated by an arrow below the feet.
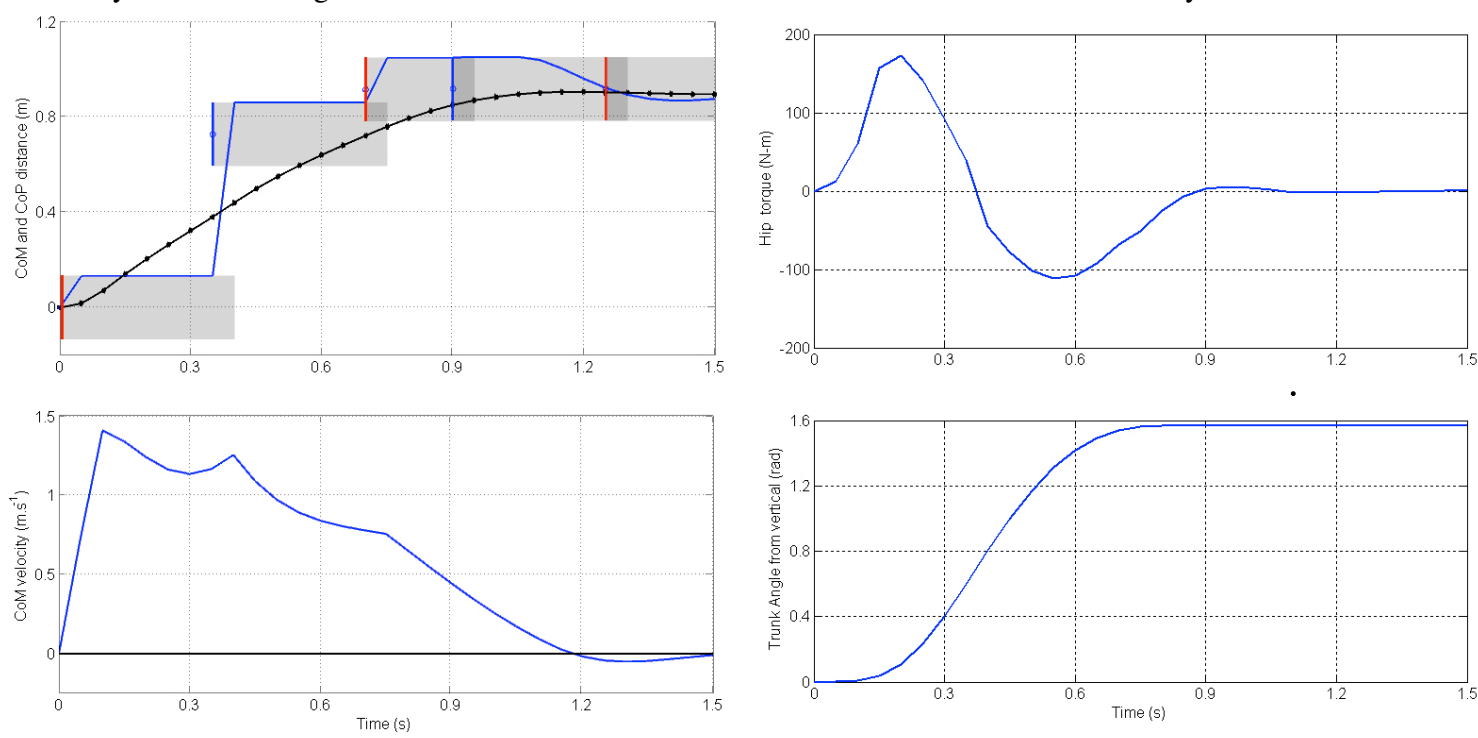

Fig. 5: Evolution with time of the positions of the CoM in black and CoP in blue during a balance recovery simulation (top left). Red and blue vertical segments precise the rhythm and positions of the steps, the gray zone emphasizing the support polygon on the ground. Corresponding evolution of CoM velocity (bottom left), upper body rotational acceleration (top right) and position (bottom right). 
Table II. The resulting balance recovery behavior can be seen in Figure \$, with corresponding plots displayed in Figure 5 . The system recovers successfully and stops completely after 2 steps of durations 0.35 and $0.3 \mathrm{~s}$, followed by a final grouping of the feet. The trunk accelerates forward during the first step to absorb the perturbation, then decelerates to avoid exceeding its maximum angle. The CoP remains most of the time at the toes during the recovery phase for maximal CoM deceleration, and goes back to the middle of the feet at the end of the motion. All in all, our scheme demonstrates a stable recovery behavior combining all 3 strategies naturally and efficiently.

Note that the final position is with a trunk bent forward close to its maximum angle $(\pi / 2)$ since the goal of the controller (21) is only to decelerate the CoM and trunk rotation, without specifying any desired final position and angle.

\section{CONCLUSion}

We presented in this article an MPC scheme that demonstrates biped balance recovery response to external perturbations by intelligently using the 3 basic strategies observed in humans. We showed how different strategies can be effectively regulated by adjusting relative weight coefficient values in the optimization criterion. In addition, the step durations were also optimized by simply penalizing the acceleration of the swing foot. Finally the simulation result demonstrated a stable recovery response to a large external disturbance to the standing posture. Future work is aimed at extending this scheme to tackle external perturbations during walking and the implementation of these ideas on a real robot.

\section{REFERENCES}

[1] F. B. Horak and L. M. Nashner, "Central programming of postural movements: adaptation to altered support-surface configurations," Journal of Neurophysiology, vol. 55, no. 6, pp. 1369-1381, 1986.

[2] L. M. Nashner and G. McCollum, "The organization of human postural movements: A formal basis and experimental synthesis," Behavioral and Brain Sciences, vol. 8, pp. 135-150, 1985.

[3] B. Maki and W. McIlroy, "The role of limb movements in maintaining upright stance: the" change-in-support" strategy," Physical Therapy, vol. 77, no. 5, p. 488, 1997.

[4] S. Park, F. Horak, and A. Kuo, "Postural feedback responses scale with biomechanical constraints in human standing," Experimental Brain Research, vol. 154, no. 4, pp. 417-427, 2004.

[5] J. Pratt, J. Carff, S. Drakunov, and A. Goswami, "Capture point: A step toward humanoid push recovery," in Proceedings of the 2006 6th IEEE-RAS International Conference on Humanoid Robots, 2006, pp. 200-207.

[6] S.-H. Hyon, R. Osu, and Y. Otaka, "Integration of multi-level postural balancing on humanoid robots," in IEEE International Conference on Robotics and Automation, 2009.

[7] K. Hirai, M. Hirose, Y. Haikawa, and T. Takenaka, "The development of honda humanoid robot," in Proc. IEEE International Conference on Robotics and Automation, vol. 2, 1998, pp. 1321-1326 vol.2.

[8] K. Nishiwaki, S. Kagami, Y. Kuniyoshi, M. Inaba, and H. Inoue, "Online generation of humanoid walking motion based on a fast generation method of motion pattern that follows desired zmp," in IEEE/RSJ International Conference on Intelligent Robots and Systems, vol. 3. IEEE, 2002, pp. 2684-2689.

[9] P.-B. Wieber, "Trajectory free linear model predictive control for stable walking in the presence of strong perturbations," in IEEE-RAS International Conference on Humanoid Robots, 2006.
[10] T. Koolen, T. D. Boer, J. Rebula, A. Goswami, and J. Pratt, "Capturability-based analysis and control of legged locomotion, part 1: Theory and application to three simple gait models," International Journal of Robotics Research, vol. 31, no. 9, p. 1094Ü1113, 2012.

[11] S.-K. Yun and A. Goswami, "Momentum-based reactive stepping controller on level and non-level ground for humanoid robot push recovery," in Proceedings of IEEE/RSJ International Conference on Intelligent Robots and Systems, 2011.

[12] D. L. Wight, E. G. Kubica, and D. W. L. Wang, "Introduction of the foot placement estimator: A dynamic measure of balance for bipedal robotics," Journal of Computational and Nonlinear Dynamics, vol. 3, p. $11009,2008$.

[13] T. Sugihara, "Standing stabilizability and stepping maneuver in planar bipedalism based on the best com-zmp regulator," in IEEE International Conference on Robotics and Automation, 2009.

[14] J. Urata, K. Nshiwaki, Y. Nakanishi, K. Okada, S. Kagami, and M. Inaba, "Online decision of foot placement using singular lq preview regulation," in IEEE-RAS International Conference on Humanoid Robots, 2011.

[15] A. Herdt, H. Diedam, P. Wieber, D. Dimitrov, K. Mombaur, and M. Diehl, "Online walking motion generation with automatic foot step placement," Advanced Robotics, vol. 24, pp. 719-737, 2010.

[16] C. Atkeson and B. Stephens, "Multiple balance strategies from one optimization criterion," in 7th IEEE-RAS International Conference on Humanoid Robots, 2007, pp. 57-64.

[17] Y. Kanamiya, S. Ota, and D. Sato, "Ankle and hip balance control strategies with transitions," in Robotics and Automation (ICRA), 2010 IEEE International Conference on, may 2010, pp. $3446-3451$.

[18] D. Nenchev and A. Nishio, "Experimental validation of ankle and hip strategies for balance recovery with a biped subjected to an impact," in IEEE/RSJ International Conference on Intelligent Robots and Systems (IROS), 29 2007-nov. 2 2007, pp. 4035 -4040.

[19] K. Yin and M. van de Panne, "Omnidirectional humanoid balance control: Multiple strategies for reacting to a push," UBC, CS Technical Report 2006-11, 2006.

[20] T. Takenaka, T. Matsumoto, T. Yoshiike, T. Hasegawa, S. Shirokura, H. Kaneko, and A. Orita, "Real time motion generation and control for biped robot -4th report: Integrated balance control-," in IEEE/RSJ International Conference on Intelligent Robots and Systems, 2009.

[21] S. Kajita and K. Tani, "Study of dynamic biped locomotion on rugged terrain-derivation and application of the linear inverted pendulum mode," IEEE International Conference on Robotics and Automation, vol. 2, pp. $1405-1411$ vol.2, apr 1991.

[22] T. Komura, H. Leung, S. Kudoh, and J. Kuffner, "A feedback controller for biped humanoids that can counteract large perturbations during gait," in Proceedings of the 2005 IEEE International Conference on Robotics and Automation (ICRA). IEEE, 2005, pp. 1989-1995.

[23] S. Kajita, F. Kanehiro, K. Kaneko, K. Fujiwara, K. Harada, K. Yokoi, and H. Hirukawa, "Biped walking pattern generation by using preview control of zero-moment point," in IEEE International Conference on Robotics and Automation, vol. 2. Citeseer, 2003, pp. 1620-1626.

[24] A. Herdt, N. Perrin, and P. Wieber, "Walking without thinking about it," in IEEE/RSJ International Conference on Intelligent Robots and Systems (IROS) 2010. IEEE, 2010, pp. 190-195.

[25] D. Winter, Biomechanics and Motor Control of Human Movement. Wiley, New York, 1990.

[26] D. Chaffin and G. Andersson, Occupational Biomechanics, 4th ed. New York, NY: J. Wiley \& Sons, 1984.

[27] H. Diedam, D. Dimitrov, P. Wieber, K. Mombaur, and M. Diehl, "Online walking gait generation with adaptive foot positioning through linear model predictive control," in Proceedings of the IEEE/RSJ International Conference on Intelligent Robots and Systems, 2008.

[28] H. Diedam, "Fast nmpc algorithms for humanoid robot walking," Master's thesis, University of Heidelberg, 2009.

[29] J. Doke and A. Kuo, "Energetic cost of producing cyclic muscle force, rather than work, to swing the human leg," Journal of Experimental Biology, vol. 210, no. 13, pp. 2390-2398, 2007.

[30] M. Zarrugh, F. Todd, and H. Ralston, "Optimization of energy expenditure during level walking," European Journal of Applied Physiology and Occupational Physiology, vol. 33, no. 4, pp. 293-306, 1974.

[31] M. Diehl, "Real-time optimization for large scale nonlinear processes," Ph.D. dissertation, University of Heidelberg, 2001. 